\title{
On the Paired-Domination Subdivision Number of Trees
}

\author{
Shouliu Wei ${ }^{1} \mathbb{C}$, Guoliang Hao ${ }^{2} \mathbb{C}$, Seyed Mahmoud Sheikholeslami ${ }^{3, *} \mathbb{C}$, Rana Khoeilar ${ }^{3}$ and Hossein Karami ${ }^{3}$ \\ 1 College of Mathematics and Data Science, Minjiang University, Fuzhou 350108, China; wslwillow@126.com \\ 2 College of Science, East China University of Technology, Nanchang 330013, China; guolianghao@ecut.edu.cn \\ 3 Department of Mathematics, Azarbaijan Shahid Madani University, Tabriz 51368, Iran; \\ khoeilar@azaruniv.ac.ir (R.K.); h.karami@azaruniv.ac.ir (H.K.) \\ * Correspondence: s.m.sheikholeslami@azaruniv.ac.ir
}

check for updates

Citation: Wei, S.; Hao, G.;

Sheikholeslami, S.M.; Khoeilar, R.; Karami, H. On the PairedDomination Subdivision Number of Trees. Mathematics 2021, 9, 1135. https://doi.org/10.3390/math9101135

Academic Editor: Sergey Kitaev and Frank Werner

Received: 12 April 2021

Accepted: 14 May 2021

Published: 17 May 2021

Publisher's Note: MDPI stays neutral with regard to jurisdictional claims in published maps and institutional affiliations.

Copyright: (c) 2021 by the authors. Licensee MDPI, Basel, Switzerland. This article is an open access article distributed under the terms and conditions of the Creative Commons Attribution (CC BY) license (https:// creativecommons.org/licenses/by/ $4.0 /)$.

\begin{abstract}
A paired-dominating set of a graph $G$ without isolated vertices is a dominating set of vertices whose induced subgraph has perfect matching. The minimum cardinality of a paireddominating set of $G$ is called the paired-domination number $\gamma_{p r}(G)$ of $G$. The paired-domination subdivision number $\operatorname{sd}_{\gamma_{p r}}(G)$ of $G$ is the minimum number of edges that must be subdivided (each edge in $G$ can be subdivided at most once) in order to increase the paired-domination number. Here, we show that, for each tree $T \neq P_{5}$ of order $n \geq 3$ and each edge $e \notin E(T), \operatorname{sd}_{\gamma_{p r}}(T)+\operatorname{sd}_{\gamma_{p r}}(T+e) \leq$ $n+2$.
\end{abstract}

Keywords: paired-domination number; paired-domination subdivision number

\section{Introduction}

Let $G=(V, E)$ be a simple connected graph with vertex set $V=V(G)$ and edge set $E(G)=E$ and let $n=|V|$. For any vertex $u \in V(G)$, the open neighborhood of $u$ is the set $N(u)=N_{G}(u)=\{v \in V(G) \mid u v \in E(G)\}$, and the closed neighborhood of $u$ is the set $N[u]=N_{G}[u]=\{u\} \cup N_{G}(u)$. The degree of a vertex $u$ is $\operatorname{deg}(u)=\operatorname{deg}_{G}(u)=\left|N_{G}(u)\right|$. A vertex of degree one is called a leaf and its neighbor is called a stem. A stem is said to be strong if it is adjacent to at least two leaves.

Throughout this paper, when an edge $e=u v$ is subdivided, $w_{e}=w_{u v}$ denotes the subdivision vertex for $e$. For a set $F \subseteq E(G), G_{F}$ denotes the graph obtained from $G$ by subdividing every edge in $F$ (note that we have $w_{e} \neq w_{f}$ for any $e, f \in F$ with $e \neq f$ ). The length of a shortest $(u, v)$-path in a graph $G$ is the distance between $u$ and $v$, and is written $d_{G}(u, v)$ or simply $d(u, v)$ if $G$ is clear from context. The maximum distance among all pairs of vertices in $G$ is called the diameter of $G$, written $\operatorname{diam}(G)$. A diametral path of $G$ is a path of $G$ with the length $\operatorname{diam}(G)$.

A subset $S$ of $V$ is a dominating set of $G$ if every vertex in $V-S$ is adjacent to a vertex in $S$. A paired-dominating set (PD-set) of $G$ is a subset $S$ of $V$ if $S$ is a dominating set and the subgraph induced by $S$ contains a perfect matching. The minimum cardinality of a PD-set of $G$ is the paired-domination number $\gamma_{p r}(G)$. If $S$ is a PD-set with a perfect matching $M$ and $u v \in M$, then $u$ and $v$ are said to be partners (or paired) in $S$. We call a PD-set of minimum cardinality a $\gamma_{p r}(G)$-set. Since the end vertices of any maximal matching in $G$ form a PD-set, every graph $G$ without isolated vertices has a PD-set. Haynes and Slater [1] introduced the Paired-domination, which has been studied, for example, in [2-6]. For more details on paired-domination, we refer the reader to [7].

As good models of many practical problems, graphs sometimes have to be changed to adapt the changes in reality. Thus, we must pay attention to the change of the graph parameters under graph modifications, such as the deletion of vertices, deletion or addition of edges, and subdivision of edges. Velammal [8] was the first to study the domination subdivision number of a graph $G$ defined to be the minimum number of edges that must be subdivided (each edge in $G$ is subdivided at most once) to increase the domination number. Since then, subdivision parameters have been studied by several authors (see [9-15]). 
In this paper, we study the paired-domination subdivision number of trees, which was introduced by Favaron et al. in [16] and has been further studied in [17-21]. The paireddomination subdivision number $\operatorname{sd}_{\gamma_{p r}}(G)$ of a graph $G$ is the minimum number of edges that must be subdivided (where each edge in $G$ can be subdivided at most once) in order to increase the paired-domination number of $G$.

If $G$ is a connected graph of order at least 3, Favaron et al. [16] asked whether it is true that for any edge $e \notin E(G), \operatorname{sd}_{\gamma_{p r}}(G+e) \leq \operatorname{sd}_{\gamma_{p r}}(G)$. Egawa et al. [18] gave a negative answer to this question. However, they proved the question in the affirmative if the following additional condition is added: $\gamma_{p r}(G+e)<\gamma_{p r}(G)$ for every $e \notin E(G)$. Recently, Hao et al. [19] showed that for any graph $G$ without isolated vertices and different from $m K_{2}$, and for any edge $e \notin E(G), \operatorname{sd}_{\gamma_{p r}}(G+e) \leq \operatorname{sd}_{\gamma_{p r}}(G)+2 \Delta(G)$.

Our aim in this paper is to further study paired-domination subdivision number and show that for each tree $T \neq P_{5}$ of order $n \geq 3$ and each edge $e \notin E(T), \operatorname{sd}_{\gamma_{p r}}(T)+$ $\mathrm{sd}_{\gamma_{p r}}(\mathrm{~T}+\mathrm{e}) \leq n+2$.

We close this section by recalling some useful results.

Proposition 1 ([16]). Let $G$ be a connected graph of order $n \geq 3$ and let $G^{\prime}$ be obtained from $G$ by subdividing the edge $e=u v \in E(G)$. Then, $\gamma_{p r}\left(G^{\prime}\right) \geq \gamma_{p r}(G)$.

Proposition 2 ([20]). For every connected graph $G$ of order $n \geq 3, \operatorname{sd}_{\gamma_{p r}}(G) \leq n-1$.

Proposition 3 ([18]). For any graph $G$ with no isolated vertex and any $u v \notin E(G), \gamma_{p r}(G)=$ $\gamma_{p r}(G+u v)$ or $\gamma_{p r}(G)=\gamma_{p r}(G+u v)+2$.

Proposition 4 ([18]). For any connected graph $G$ of order at least 3 and uv $\notin E(G)$ with $\gamma_{p r}(G+u v)<\gamma_{p r}(G), \operatorname{sd}_{\gamma_{p r}}(G+u v) \leq \operatorname{sd}_{\gamma_{p r}}(G)$.

Proposition 5 ([16]). If $G$ contains either adjacent stems or a strong stem, then $\operatorname{sd}_{\gamma_{p r}}(G) \leq 2$.

Proposition 6 ([16]). For any connected graph $G$ containing a path $v_{1} v_{2} v_{3} v_{4} v_{5}$ such that $\operatorname{deg}\left(v_{2}\right)=\operatorname{deg}\left(v_{3}\right)=\operatorname{deg}\left(v_{4}\right)=2, \operatorname{sd}_{\gamma_{p r}}(G) \leq 4$.

Proposition 7 ([16]). For $n \geq 3$,

$$
\operatorname{sd}_{\gamma_{p r}}\left(P_{n}\right)=\operatorname{sd}_{\gamma_{p r}}\left(C_{n}\right)=\left\{\begin{array}{llll}
1, & \text { if } & n \equiv 0 & (\bmod 4) \\
4, & \text { if } & n \equiv 1 & (\bmod 4) \\
3, & \text { if } & n \equiv 2 & (\bmod 4) \\
2, & \text { if } & n \equiv 3 & (\bmod 4)
\end{array}\right.
$$

Proposition 8 ([18]). If a tree $T$ contains a path $v_{1} v_{2} v_{3} v_{4}$ in which $\operatorname{deg}\left(v_{1}\right)=1$ and $\operatorname{deg}\left(v_{i}\right)=2$ for $i=2,3$, then $\operatorname{sd}_{\gamma_{p r}}(T) \leq 4$.

Proposition 9 ([19]). For any isolated-free graph $G$ different from $m K_{2}$ and any uv $\notin E(G)$ satisfying that $u$ or $v$ is a stem,

$$
\operatorname{sd}_{\gamma_{p r}}(G+u v) \leq \operatorname{sd}_{\gamma_{p r}}(G)+2
$$

For any positive integer $m \geq 1$, let $S_{m}$ be the healthy spider obtained from the complete bipartite graph $K_{1, m}$ by subdividing every edge. Therefore $V\left(S_{m}\right)=\left\{u_{1}^{i}, u_{2}^{i} \mid 1 \leq i \leq\right.$ $m\} \cup\{x\}$ and $E\left(S_{m}\right)=\left\{x u_{2}^{i}, u_{2}^{i} u_{1}^{i} \mid 1 \leq i \leq m\right\}$ (see Figure 1). The vertex $x$ is called the center of $S_{m}$. Let $T_{1, m}$ be the tree obtained from the disjoint union of two copies of the healthy spider $S_{m}$ centered at $x, y$, by joining $x$ and $y$ (see Figure 2). Observe that $n\left(T_{1, m}\right)=4 m+2, \gamma_{p r}\left(T_{1, m}\right)=4 m$ and $\operatorname{sd}_{\gamma_{p r}}\left(T_{1, m}\right)=2 m+1$. 


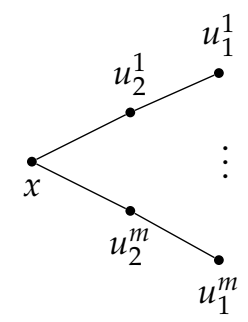

Figure 1. A healthy spider $S_{m}$.

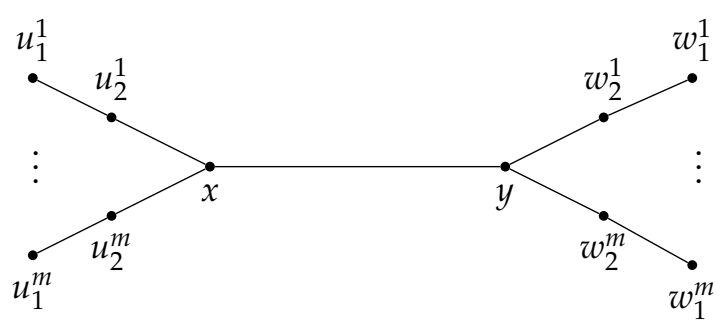

Figure 2. A tree $T_{1, m}$ with $\operatorname{sd}_{\gamma_{p r}}\left(T_{1, m}\right)=n\left(T_{1, m}\right) / 2$.

Let $T_{2, m}$ be the tree obtained from $T_{1, m}$ by subdividing the edge $x y$ with a subdivision vertex $u$ and adding a new vertex $v$ and a new edge $u v$ (Figure 3). Observe that $n\left(T_{2, m}\right)=$ $4 m+4, \gamma_{p r}\left(T_{2, m}\right)=4 m+2$, and $\operatorname{sd}_{\gamma_{p r}}\left(T_{2, m}\right)=2 m+2$.

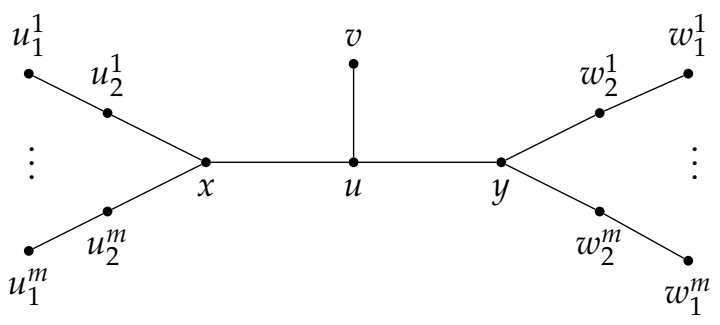

Figure 3. A tree $T_{2, m}$ with $\operatorname{sd}_{\gamma_{p r}}\left(T_{2, m}\right)=n\left(T_{2, m}\right) / 2$.

Now let $\mathcal{T}=\left\{K_{1,3}\right\} \cup\left\{S_{m}, T_{1, m}, T_{2, m} \mid m \geq 1\right\}$.

Proposition 10 ([18]). For any tree $T$ of order $n \geq 3, \operatorname{sd}_{\gamma_{p r}}(T) \geq n / 2$ if and only if $T \in \mathcal{T}$.

Proposition 11 ([20]). For any tree $T$ of order $n \geq 4$ different from a healthy spider, $\operatorname{sd}_{\gamma_{p r}}(T) \leq \frac{n}{2}$.

Combining Propositions 10 and 11, we have the following corollary.

Corollary 1. Let $T$ be a tree of order $n \geq 4$ different from a healthy spider. Then, $\operatorname{sd}_{\gamma_{p r}}(T) \leq \frac{n}{2}$ with equality if and only if $T \in\left\{K_{1,3}, T_{1, m}, T_{2, m} \mid m \geq 1\right\}$.

\section{Main Result}

Our aim in this section is to prove that, for each tree $T \neq P_{5}$ with $n \geq 3$ vertices and any edge $e \notin E(T), \operatorname{sd}_{\gamma_{p r}}(T)+\operatorname{sd}_{\gamma_{p r}}(T+e) \leq n+2$. First, we consider trees with diameter four and five.

Lemma 1. Let $T$ be a tree of order $n \geq 6$ with $\operatorname{diam}(T)=4$ and let $e=x y \notin E(G)$. Then,

$$
\operatorname{sd}_{\gamma_{p r}}(T)+\operatorname{sd}_{\gamma_{p r}}(T+e) \leq n+2 .
$$

Proof. If $T$ has a strong stem or adjacent stems, then by Propositions 2 and 5 we have $\operatorname{sd}_{\gamma_{p r}}(T)+\operatorname{sd}_{\gamma_{p r}}(T+e) \leq n+1$. Suppose next that $T$ has no adjacent stems and no strong 
stem. Then, $T$ is a healthy spider. Let $V(T)=\left\{v, v_{i}, u_{i} \mid 1 \leq i \leq t\right\}$ and let $E(T)=$ $\left\{v v_{i}, v_{i} u_{i} \mid 1 \leq i \leq t\right\}$. Since $n \geq 6$, we have $t \geq 3$. We consider the four cases.

Case 1. Both $x$ and $y$ are leaves.

Without a loss of generality, assume that $x=u_{1}$ and $y=u_{2}$. Observe that $\gamma_{p r}(T+e)=$ $2 t-2$. Let $T^{\prime}$ be obtained from $T+e$ by subdividing the edges $v v_{1}, v v_{2}, v_{1} u_{1}$ with the vertices $w_{1}, w_{2}$, and $w_{3}$, respectively. We show that $\gamma_{p r}\left(T^{\prime}\right) \geq 2 t$. Let $D$ be a $\gamma_{p r}\left(T^{\prime}\right)$-set. If $v \notin$ $D$, then we must have $v_{i}, u_{i} \in D$ for each $3 \leq i \leq t$ and $\left|D \cap\left\{v_{1}, v_{2}, u_{1}, u_{2}, w_{1}, w_{2}, w_{3}\right\}\right| \geq 4$, which leads to $\gamma_{p r}\left(T^{\prime}\right)=|D| \geq 2 t$. Assume now that $v \in D$. If $v$ is paired with $w_{1}$ or $w_{2}$, then we must have $v_{i}, u_{i} \in D$ for each $3 \leq i \leq t$ and $\left|D \cap\left\{v, v_{1}, v_{2}, u_{1}, u_{2}, w_{1}, w_{2}, w_{3}\right\}\right| \geq 4$ implying that $\gamma_{p r}\left(T^{\prime}\right)=|D| \geq 2 t$. Assume next that $v$ is not paired with $w_{1}$ or $w_{2}$. Without a loss of generality, assume that $v$ is paired with $v_{3}$. Thus, $v_{i}, u_{i} \in D$ for each $4 \leq i \leq t$ and $\left|D \cap\left\{v_{1}, v_{2}, u_{1}, u_{2}, w_{1}, w_{2}, w_{3}\right\}\right| \geq 4$ yielding $\gamma_{p r}\left(T^{\prime}\right)=|D| \geq 2 t$. Thus, $\operatorname{sd}_{\gamma_{p r}}(T+e) \leq 3$ and, by Proposition 2, we obtain $\operatorname{sd}_{\gamma_{p r}}(T)+\operatorname{sd}_{\gamma_{p r}}(T+e) \leq n+2$.

Case 2. $x$ is a leaf and $y$ is a stem.

Without a loss of generality, assume that $x=u_{1}$ and $y=v_{2}$. Then, it is clear that $\gamma_{p r}(T+e)=2 t-2$. Let $T^{\prime}$ be obtained from $T+e$ by subdividing the edges $v_{2} u_{2}$ and $e$ with the vertices $w_{1}$ and $w_{2}$, respectively. As in Case 1 , we can see that $\gamma_{p r}\left(T^{\prime}\right) \geq 2 t$. Hence, $\operatorname{sd}_{\gamma_{p r}}(T+e) \leq 2$ and, by Proposition 2, we have $\operatorname{sd}_{\gamma_{p r}}(T)+\operatorname{sd}_{\gamma_{p r}}(T+e) \leq n+1$.

Case 3. $x$ is a leaf and $y=v$.

Without a loss of generality, assume that $x=u_{1}$. Clearly $\gamma_{p r}(T+e)=2 t-2$. Let $T^{\prime}$ be obtained from $T+e$ by subdividing the edges $u_{1} v_{1}$ with $w_{1}$. We show that $\gamma_{p r}\left(T^{\prime}\right) \geq 2 t$. Let $D$ be a $\gamma_{p r}\left(T^{\prime}\right)$-set. To paired-dominate $u_{2}, \ldots, u_{t}$, we must have $\mid D \cap\left\{v, u_{i}, v_{i} \mid 2 \leq\right.$ $i \leq t\} \mid \geq 2 t-2$ and to paired-dominate $u_{1}$ we must have $\left|D \cap\left\{u_{1}, v_{1}, w_{1}\right\}\right| \geq 1$. Since $|D|$ is even, we obtain $\gamma_{p r}\left(T^{\prime}\right) \geq 2 t$. Hence, $\operatorname{sd}_{\gamma_{p r}}(T+e)=1$ and, by Proposition 2, we have $\operatorname{sd}_{\gamma_{p r}}(T)+\operatorname{sd}_{\gamma_{p r}}(T+e) \leq n$.

Case 4. Both $x$ and $y$ are stems.

In this case, $T^{\prime}$ has adjacent stems, and we deduce from Propositions 2 and 5 that $\mathrm{sd}_{\gamma_{p r}}(T)+\operatorname{sd}_{\gamma_{p r}}(T+e) \leq n+1$.

Lemma 2. Let $T$ be a tree of order $n$ with $\operatorname{diam}(T)=5$ and let $e=x y \notin E(G)$. Then,

$$
\operatorname{sd}_{\gamma_{p r}}(T)+\operatorname{sd}_{\gamma_{p r}}(T+e) \leq n+2 .
$$

Proof. As in the proof of Lemma 1, we may assume that $T$ has no strong stem and no adjacent stems. Let $u_{1} w_{1} v_{1} v_{2} x_{1} y_{1}$ be a diametral path in $T$. By the assumption, the components of $T-v_{1} v_{2}$ are $P_{3}$ or a healthy spider. It is easy to see that $\gamma_{p r}(T)=n-2$. Let $T_{1}$ and $T_{2}$ be the components of $T-v_{1} v_{2}$ containing $v_{1}$ and $v_{2}$, respectively. If $T=P_{6}$, then we deduce from Propositions 2 and 7 that $\operatorname{sd}_{\gamma_{p r}}(T)+\operatorname{sd}_{\gamma_{p r}}(T+e) \leq n+2$. Assume that $T \neq P_{6}$. Suppose that $\operatorname{deg}\left(v_{2}\right) \geq 3$. If $\operatorname{deg}\left(v_{1}\right)=2$, then it is easy to see that subdividing the edges $u_{1} w_{1}, w_{1} v_{1}, v_{1} v_{2}$ increases the paired-domination number of $T$ and so $\operatorname{sd}_{\gamma_{p r}}(T) \leq 3$ and the result follows by Proposition 2. Hence, we assume that $\operatorname{deg}\left(v_{1}\right) \geq 3$. Let $V\left(T_{1}\right)=$ $\left\{v_{1}, w_{i}, u_{i} \mid 1 \leq i \leq t\right\}, E\left(T_{1}\right)=\left\{v_{1} w_{i}, w_{i} u_{i} \mid 1 \leq i \leq t\right\}, V\left(T_{2}\right)=\left\{v_{2}, x_{i}, y_{i} \mid 1 \leq i \leq s\right\}$ and let $E\left(T_{2}\right)=\left\{v_{2} x_{i}, x_{i} y_{i} \mid 1 \leq i \leq s\right\}$. We consider the four cases.

Case 1. Both $x$ and $y$ are leaves.

If $x$ and $y$ are the leaves of $T_{1}$ (resp., $T_{2}$ ), then, clearly, $\gamma_{p r}(T+x y)<\gamma_{p r}(T)$ and by Propositions 4 and 11, we have $\operatorname{sd}_{\gamma_{p r}}(T)+\operatorname{sd}_{\gamma_{p r}}(T+e) \leq n$. If $x$ is a leaf of $T_{1}$ and $y$ is a leaf of $T_{2}$, then, we deduce from Propositions 6 and 11 that $\operatorname{sd}_{\gamma_{p r}}(T)+\operatorname{sd}_{\gamma_{p r}}(T+e) \leq$ $n / 2+4<n+2$.

Case 2. $x$ is a leaf and $y$ is a stem. 
Assume first that $x, y$ are the vertices of $T_{1}$. We may assume that $x=u_{1}$ and $y=w_{2}$. Then, the set $D=\left\{v_{1}, w_{2}\right\} \cup\left\{w_{i}, u_{i} \mid 3 \leq i \leq t\right\} \cup\left\{x_{j}, y_{j} \mid 1 \leq j \leq s\right\}$ is a paireddominating set of $T+e$ of size $n-4$ and so $\gamma_{p r}(T+e)<n-2=\gamma_{p r}(T)$. If $x$ and $y$ are the vertices of $T_{2}$, then similarly we have $\gamma_{p r}(T+e)<\gamma_{p r}(T)$. Assume second that $x \in V\left(T_{1}\right)$ and $y \in V\left(T_{2}\right)$. Without a loss of generality, we can suppose that $x=u_{1}$ and $y=x_{1}$. Then, the set $\left\{v_{1}, v_{2}, x_{1}, w_{2}\right\} \cup\left\{w_{i}, u_{i} \mid 3 \leq i \leq t\right\} \cup\left\{x_{j}, y_{j} \mid 2 \leq j \leq s\right\}$ is a paired-dominating set of $T+e$ with cardinality $n-4$, and thus $\gamma_{p r}(T+e)<n-2=\gamma_{p r}(T)$. Applying Propositions 4 and 11 we obtain $\operatorname{sd}_{\gamma_{p r}}(T)+\operatorname{sd}_{\gamma_{p r}}(T+e) \leq n$.

Case 3. $x$ is a leaf and $y \in\left\{v_{1}, v_{2}\right\}$.

Without loss of generality, we may assume that $x=u_{1}$. If $y=v_{1}$, then the set $\left\{v_{1}, w_{2}\right\} \cup\left\{w_{i}, u_{i} \mid 3 \leq i \leq t\right\} \cup\left\{x_{j}, y_{j} \mid 1 \leq j \leq s\right\}$ is a paired-dominating set of $T+e$ of size $n-4$ and so $\gamma_{p r}(T+e)<n-2=\gamma_{p r}(T)$. If $y=v_{2}$, then the set $\left\{v_{1}, v_{2}, w_{2}, x_{1}\right\} \cup$ $\left\{w_{i}, u_{i} \mid 3 \leq i \leq t\right\} \cup\left\{x_{j}, y_{j} \mid 2 \leq j \leq s\right\}$ is a paired-dominating set of $T+e$ of size $n-4$, and thus $\gamma_{p r}(T+e)<n-2=\gamma_{p r}(T)$. Using Propositions 4 and 11, we obtain $\operatorname{sd}_{\gamma_{p r}}(T)+\operatorname{sd}_{\gamma_{p r}}(T+e) \leq n$.

Case 4. Both $x$ and $y$ are stems.

In this case, $T^{\prime}+x y$ has adjacent stems, and we deduce from Propositions 2 and 5 that $\operatorname{sd}_{\gamma_{p r}}(T)+\operatorname{sd}_{\gamma_{p r}}(T+e) \leq n+1$.

Theorem 1. Let $T$ be a tree different from $P_{5}$ of order $n \geq 3$ and let $e=x y \notin E(G)$. Then,

$$
\operatorname{sd}_{\gamma_{p r}}(T)+\operatorname{sd}_{\gamma_{p r}}(T+e) \leq n+2 .
$$

Proof. If $T$ has a strong stem or adjacent stems, then, by Propositions 2 and 5, we have $\operatorname{sd}_{\gamma_{p r}}(T)+\operatorname{sd}_{\gamma_{p r}}(T+e) \leq n+1$. Hence, we assume that $T$ has no strong stem and no adjacent stems. It follows that $\operatorname{diam}(T) \geq 4$. According to Lemmas 1 and 2, we may assume that $\operatorname{diam}(T) \geq 6$. If $x$ or $y$ is a stem, then by Propositions 9 and 11 , we have $\operatorname{sd}_{\gamma_{p r}}(T)+\operatorname{sd}_{\gamma_{p r}}(T+e) \leq n+2$. Hence, we assume that neither $x$ nor $y$ is a stem. If $x$ (resp., $y$ ) is a leaf with support vertex $x^{\prime}$ (resp., $y^{\prime}$ ) of degree 2, respectively, then for $x^{\prime \prime} \in N\left(x^{\prime}\right)-\{x\}, x^{\prime \prime} x^{\prime} x y y^{\prime}$ is a path in $T$ with $\operatorname{deg}\left(x^{\prime}\right)=\operatorname{deg}(x)=\operatorname{deg}(y)=2$, and therefore by Propositions 6 and 11, we have $\operatorname{sd}_{\gamma_{p r}}(T)+\operatorname{sd}_{\gamma_{p r}}(T+e) \leq n / 2+4<n+2$. Thus, we may assume that, if both $x$ and $y$ are leaves, then the stem of $x$ or $y$ is of at least degree 3.

Let $v_{1} v_{2} \ldots v_{k}$ be a diametral path such that $\operatorname{deg}\left(v_{3}\right)$ is minimized. By assumption, the trees $T_{1}$ and $T_{2}$ (the components of $T-\left\{v_{3} v_{4}, v_{k-2} v_{k-3}\right\}$ containing $v_{3}$ and $v_{k-2}$, respectively) are $P_{3}$ or a healthy spider. Let $N\left(v_{3}\right)=\left\{v_{4}\right\} \cup\left\{u_{1}=v_{2}, u_{2}, \ldots, u_{t}\right\}$ and let $u_{i}^{\prime}$ be the leaf adjacent to $u_{i}$ for each $i$. Root $T$ is at $v_{4}$. We consider three cases.

Case 1. $x, y \notin V\left(T_{1}\right)$.

We distinguish the following subcases.

Subcase 1.1. $v_{4}$ is a stem.

By our assumption, $T$ has no strong stem. Let $w$ be a unique leaf adjacent to $v_{4}$. First, let $w \notin\{x, y\}$. Let $T^{\prime}$ be obtained from $T+e$ by subdividing the edges $v_{3} u_{i}$ and $u_{i} u_{i}^{\prime}$ with vertices $x_{i}$ and $y_{i}$, respectively for each $i$ and the edges $v_{3} v_{4}$ and $v_{4} w$ with vertices $r$ and $s$, respectively. We show that $\gamma_{p r}\left(T^{\prime}\right)>\gamma_{p r}(T+e)$. Let $P$ be a $\gamma_{p r}\left(T^{\prime}\right)$-set. To paired-dominate $u_{i}^{\prime}$, we may assume that $u_{i}, y_{i} \in P$ for each $i$. On the other hand, to paired-dominate $w$ and $v_{3}$, we must have $\left|P \cap\left\{v_{3}, v_{4}, w, r, s, x_{1}, x_{2}, \ldots, x_{t}\right\}\right| \geq 4$.

If $v_{4} \notin P$ or $v_{4} \in P$ and $v_{4}$ is paired with $r$ or $s$, then the set

$$
\left(P-\left\{v_{3}, v_{4}, w, r, s, x_{1}, x_{2}, \ldots, x_{t}, y_{1}, y_{2}, \ldots, y_{t}\right\}\right) \cup\left\{v_{3}, v_{4}, u_{1}^{\prime}, u_{2}^{\prime}, \ldots, u_{t}^{\prime}\right\}
$$

is a PD-set of $T+e$ with cardinality of less than $P$. Assume that $v_{4} \in P$ and $v_{4}$ is paired with a vertex not in $\{r, s\}$. Then, we have $\left|P \cap\left\{w, r, s, v_{3}, x_{1}, x_{2}, \ldots, x_{t}\right\}\right| \geq 4$, and clearly 
the set $\left(P-\left\{v_{3}, w, r, s, x_{1}, x_{2}, \ldots, x_{t}, y_{1}, y_{2}, \ldots, y_{t}\right\}\right) \cup\left\{u_{1}^{\prime}, u_{2}^{\prime}, \ldots, u_{t}^{\prime}\right\}$ is a PD-set of $T+e$ with cardinality of less than $P$. Since the number of subdivided edges is at most $n / 2$, we deduce from Proposition 11 that $\mathrm{sd}_{\gamma_{p r}}(T)+\operatorname{sd}_{\gamma_{p r}}(T+e) \leq n$.

Now, let $w \in\{x, y\}$. Let $N\left(v_{4}\right)=\left\{z_{1}=v_{3}, z_{2}=v_{5}, z_{3}=w, z_{4}, \ldots, z_{\ell}\right\}$. Note that each component of $(T+e)-\left\{v_{4} z_{i} \mid 1 \leq j \leq \ell\right\}$ has at least two vertices. Let $T^{\prime}$ be obtained from $T+e$ by subdividing $v_{3} u_{i}$ and $u_{i} u_{i}^{\prime}$ with vertices $x_{i}$ and $y_{i}$, respectively for each $i$, the edge $v_{4} z_{j}$ with vertex $z_{j}^{\prime}$ for each $j$, and the edge $e$ with vertex $q$. By our assumptions, it is not difficult to verify that the number of subdivided edges is at most $n / 2+2$. We next show that $\gamma_{p r}\left(T^{\prime}\right)>\gamma_{p r}(T+e)$. Let $P$ be a $\gamma_{p r}\left(T^{\prime}\right)$-set, and let $F$ be the set of all edges in $\left\{e, v_{4} z_{j} \mid 2 \leq j \leq \ell\right\}$ whose subdivision vertices are in $P$. To paired-dominate $u_{i}^{\prime}$, we may assume that $u_{i}, y_{i} \in P$ for each $i$.

On the other hand, to paired-dominate $v_{3}$, we must have $\left|P \cap\left\{v_{3}, v_{4}, z_{1}^{\prime}, x_{1}, x_{2}, \ldots, x_{t}\right\}\right|$ $\geq 2$. If $v_{4} \notin P$ or $v_{4} \in P$ and $v_{4}$ is not paired with $z_{1}^{\prime}$, then $\left|P \cap\left\{z_{1}^{\prime}, v_{3}, x_{1}, x_{2}, \ldots, x_{t}\right\}\right| \geq 2$ and the set $\left(P-\left\{v_{3}, z_{1}^{\prime}, x_{1}, x_{2}, \ldots, x_{t}, y_{1}, y_{2}, \ldots, y_{t}\right\}\right) \cup\left\{u_{1}^{\prime}, u_{2}^{\prime}, \ldots, u_{t}^{\prime}\right\}$ is a PD-set of $G_{1}$ obtained from $T+e$ by subdividing all edges in $F$ with cardinality of less than $P$. If $v_{4} \in P$ and $v_{4}$ is paired with $z_{1}^{\prime}$, then the set $\left(P-\left\{v_{4}, z_{1}^{\prime}, x_{1}, x_{2}, \ldots, x_{t}, y_{1}, y_{2}, \ldots, y_{t}\right\}\right) \cup$ $\left\{v_{3}, u_{2}^{\prime}, u_{3}^{\prime}, \ldots, u_{t}^{\prime}\right\}$ is a PD-set of $G_{2}$ obtained from $T+e$ by subdividing the edges in $F$ with cardinality of less than $P$. By Proposition 1, we have $\gamma_{p r}\left(T^{\prime}\right)>\gamma_{p r}\left(G_{i}\right) \geq \gamma_{p r}(T+e)$ for each $i \in\{1,2\}$. Therefore, in either case, we have $\operatorname{sd}_{\gamma_{p r}}(T+e) \leq n / 2+2$. It follows from Proposition 11 that $\mathrm{sd}_{\gamma_{p r}}(T)+\operatorname{sd}_{\gamma_{p r}}(T+e) \leq n+2$.

Subcase 1.2. $v_{4}$ is not a stem and there is a path $v_{4} w_{2} w_{1}$ in $T$ such that $\operatorname{deg}\left(w_{1}\right)=1$ and $\operatorname{deg}\left(w_{2}\right)=2$.

If $e$ is incident to $w_{2}$, then it follows from Propositions 9 and 11 that $\operatorname{sd}_{\gamma_{p r}}(T)+$ $\operatorname{sd}_{\gamma_{p r}}(T+e) \leq n+2$. Hence, we assume that $w_{2} \notin\{x, y\}$. First, let $w_{1} \notin\{x, y\}$. Let $T^{\prime}$ be obtained from $T+e$ by subdividing the edges $v_{3} u_{i}$ and $u_{i} u_{i}^{\prime}$ with the vertices $x_{i}$ and $y_{i}$, respectively, for each $i$ and the edges $v_{3} v_{4}$ and $v_{4} w_{2}$ with the vertices $r$ and $s$, respectively. By our assumptions, it is not difficult to verify that the number of subdivided edges is at most $n / 2$. As above, we can see that $\gamma_{p r}\left(T^{\prime}\right)>\gamma_{p r}(T+e)$, and thus $\operatorname{sd}_{\gamma_{p r}}(T+e) \leq n / 2$. We deduce from Proposition 11 that $\operatorname{sd}_{\gamma_{p r}}(T)+\operatorname{sd}_{\gamma_{p r}}(T+e) \leq n$.

Now, let $w_{1} \in\{x, y\}$. We distinguish the following situations.

(1.2.1) $v_{4} \notin\{x, y\}$.

Let $N\left(v_{4}\right)=\left\{z_{1}=v_{3}, z_{2}=v_{5}, z_{3}=w_{2}, z_{4}, \ldots, z_{\ell}\right\}$. Note that each component of $(T+e)-\left\{v_{4} z_{i} \mid 1 \leq j \leq \ell\right\}$ has order at least two. Let $T^{\prime}$ be obtained from $T+e$ by subdividing the edges $v_{3} u_{i}$ and $u_{i} u_{i}^{\prime}$ with the vertices $x_{i}$ and $y_{i}$, respectively, for each $i$, the edge $v_{4} z_{j}$ with vertex $z_{j}^{\prime}$ for each $j$, and the edges $w_{2} w_{1}$ and $e$ with the vertices $q_{1}$ and $q_{2}$, respectively. By our assumptions, it is not difficult to verify that the number of subdivided edges is at most $(n+3) / 2$. We show that $\gamma_{p r}\left(T^{\prime}\right)>\gamma_{p r}(T+e)$. Let $P$ be a $\gamma_{p r}\left(T^{\prime}\right)$-set, and let $F$ be the set of all edges in $\left\{w_{1} w_{2}, e, v_{4} z_{j} \mid 2 \leq j \leq \ell\right\}$ whose subdivision vertices are in $P$. To paired-dominate $u_{i}^{\prime}$, we may assume that $u_{i}, y_{i} \in P$ for each $i$. On the other hand, to paired-dominate $v_{3}$, we must have $\left|P \cap\left\{v_{4}, z_{1}^{\prime}, v_{3}, x_{1}, x_{2}, \ldots, x_{t}\right\}\right| \geq 2$.

If $v_{4} \notin P$ or $v_{4} \in P$ and $v_{4}$ is not paired with $z_{1}^{\prime}$, then $\left|P \cap\left\{v_{3}, z_{1}^{\prime}, x_{1}, x_{2}, \ldots, x_{t}\right\}\right| \geq 2$, and the set $\left(P-\left\{v_{3}, z_{1}^{\prime}, x_{1}, x_{2}, \ldots, x_{t}, y_{1}, y_{2}, \ldots, y_{t}\right\}\right) \cup\left\{u_{1}^{\prime}, u_{2}^{\prime}, \ldots, u_{t}^{\prime}\right\}$ is a PD-set of $G_{3}$ obtained from $T+e$ by subdividing the edges $F$ with cardinality of less than $P$. If $v_{4} \in P$ and $v_{4}$ is paired with $z_{1}^{\prime}$, then the set $\left(P-\left\{v_{4}, z_{1}^{\prime}, x_{1}, x_{2}, \ldots, x_{t}, y_{1}, y_{2}, \ldots, y_{t}\right\}\right) \cup$ $\left\{v_{3}, u_{2}^{\prime}, u_{3}^{\prime}, \ldots, u_{t}^{\prime}\right\}$ is a PD-set of $G_{4}$ obtained from $T+e$ by subdividing the edges in $F$ with cardinality of less than $P$. By Proposition 1, we have $\gamma_{p r}\left(T^{\prime}\right)>\gamma_{p r}\left(G_{i}\right) \geq$ $\gamma_{p r}(T+e)$ for each $i \in\{3,4\}$. Therefore, in either case, we have $\operatorname{sd}_{\gamma_{p r}}(T+e) \leq$ $(n+3) / 2$. It follows from Proposition 11 that $\operatorname{sd}_{\gamma_{p r}}(T)+\operatorname{sd}_{\gamma_{p r}}(T+e)<n+2$.

(1.2.2) $v_{4} \in\{x, y\}$.

Then, $e=v_{4} w_{1}$. Let $N\left(v_{4}\right)=\left\{z_{1}=v_{3}, z_{2}=v_{5}, z_{3}=w_{2}, z_{4}, \ldots, z_{\ell}\right\}$, and let $T^{\prime}$ be obtained from $T+e$ by subdividing the edges $v_{3} u_{i}$ and $u_{i} u_{i}^{\prime}$ with the vertices $x_{i}$ and $y_{i}$, respectively, for each $i$, the edge $v_{4} z_{j}$ with vertex $z_{j}^{\prime}$ for each $j$, and the edges $w_{2} w_{1}$ 
and $e$. By our assumptions, it is not difficult to verify that the number of subdivided edges is at most $n / 2+2$. Using an argument similar to that described in (1.2.1), we can see that $\operatorname{sd}_{\gamma_{p r}}(T)+\operatorname{sd}_{\gamma_{p r}}(T+e) \leq n+2$.

Subcase 1.3. All children of $v_{4}$ except $v_{5}$ have depth 3 .

Considering the above cases and subcases and the choice of diametral path, we may assume that each component of $(T+e)-v_{4}$ has at least three vertices. Let $N\left(v_{4}\right)=\left\{z_{1}=\right.$ $\left.v_{3}, z_{2}=v_{5}, z_{3}, \ldots, z_{\ell}\right\}$, and let $T^{\prime}$ be obtained from $T+e$ by subdividing the edges $v_{3} u_{i}$ and $u_{i} u_{i}^{\prime}$ with the vertices $x_{i}$ and $y_{i}$, respectively, for each $i$ and the edges $v_{4} z_{j}$ with vertex $z_{j}^{\prime}$ for each $j$. By our assumptions, it is not difficult to verify that the number of subdivided edges is at most $n / 2+1$. We show that $\gamma_{p r}\left(T^{\prime}\right)>\gamma_{p r}(T+e)$. Let $P$ be a $\gamma_{p r}\left(T^{\prime}\right)$-set. To paired-dominate $u_{i}^{\prime}$, we may assume that $u_{i}, y_{i} \in P$ for each $i$. On the other hand, to paired-dominate $v_{3}$, we must have $\left|P \cap\left\{v_{3}, v_{4}, z_{1}^{\prime}, x_{1}, \ldots, x_{t}\right\}\right| \geq 2$.

Let $F$ be the set of subdivided edges incident to $v_{4}$ whose subdivision vertices belong to $P$. If $v_{4} \notin P$ or $v_{4} \in P$ and its partner is in $\left\{z_{2}^{\prime}, \ldots, z_{s}^{\prime}\right\}$, then we have $\mid P \cap$ $\left\{v_{3}, z_{1}^{\prime}, x_{1}, \ldots, x_{t}\right\} \mid \geq 2$, and the set $\left(P-\left(\left\{v_{3}, z_{1}^{\prime}\right\} \cup\left\{x_{i}, y_{i} \mid 1 \leq i \leq t\right\}\right) \cup\left\{v_{3}, u_{1}\right\} \cup\left\{u_{i}, u_{i}^{\prime} \mid\right.\right.$ $2 \leq i \leq t\}$ is a PD-set of $G_{5}$ obtained from $T+e$ by subdividing the edges of $F$ with cardinality of less than $P$.

If $v_{4} \in P$, and its partner is $z_{1}^{\prime}$, then the set $\left(P-\left(\left\{v_{4}, z_{1}^{\prime}\right\} \cup\left\{y_{i} \mid 1 \leq i \leq t\right\}\right) \cup\right.$ $\left\{v_{3}, u_{1}\right\} \cup\left\{u_{i}, u_{i}^{\prime} \mid 2 \leq i \leq t\right\}$ is a PD-set of $G_{6}$ obtained from $T+e$ by subdividing the edges of $F$ with cardinality of less than $P$. By Proposition 1, we have $\gamma_{p r}\left(T^{\prime}\right)>\gamma_{p r}\left(G_{i}\right) \geq$ $\gamma_{p r}(T+e)$ for each $i \in\{5,6\}$. Therefore, in either case, we have $\operatorname{sd}_{\gamma_{p r}}(T+e) \leq n / 2+1$. It follows from Proposition 11 that $\mathrm{sd}_{\gamma_{p r}}(T)+\mathrm{sd}_{\gamma_{p r}}(T+e) \leq n+1$.

Case 2. $x, y \in V\left(T_{1}\right)$.

By our earlier assumptions, $T$ has no strong stem, neither $x$ nor $y$ is a stem, and if both $x$ and $y$ are leaves, then the stem of $x$ or $y$ is of at least degree 3. Therefore, we may assume without a loss of generality that $x=v_{3}$ and $y=v_{1}$. Let $T^{\prime}$ be obtained from $T+e$ by subdividing the edges $v_{3} v_{2}, v_{2} v_{1}$, and $e$ by the subdivision vertices $x_{1}, x_{2}$, and $x_{3}$, respectively. We show that $\gamma_{p r}\left(T^{\prime}\right)>\gamma_{p r}(T+e)$. Let $P$ be a $\gamma_{p r}\left(T^{\prime}\right)$-set. If $v_{3} \notin P$, then we must have $\left|P \cap\left\{x_{1}, x_{2}, x_{3}, v_{1}, v_{2}\right\}\right| \geq 4$, and clearly $\left(P-\left\{x_{1}, x_{2}, x_{3}, v_{1}, v_{2}\right\}\right) \cup\left\{v_{1}, v_{2}\right\}$ is a PD-set of $T+e$ with cardinality of less than $P$.

If $v_{3} \in P$ and its partner is not $x_{1}$ or $x_{3}$, then $\left|P \cap\left\{x_{1}, x_{2}, x_{3}, v_{1}, v_{2}\right\}\right| \geq 2$, and hence $P-\left\{x_{1}, x_{2}, x_{3}, v_{1}, v_{2}\right\}$ is a PD-set of $T+e$ with cardinality of less than $P$. If $v_{3} \in P$ and its partner is $x_{1}$ or $x_{3}$, then $\left|P \cap\left\{x_{1}, x_{2}, x_{3}, v_{1}, v_{2}\right\}\right| \geq 3$, and hence $\left(P-\left\{x_{1}, x_{2}, x_{3}, v_{1}, v_{2}\right\}\right) \cup$ $\left\{v_{2}\right\}$ is a PD-set of $T+e$ with cardinality of less than $P$. Therefore, we have $\operatorname{sd}_{\gamma_{p r}}(T+e) \leq 3$. It follows from Proposition 11 that $\operatorname{sd}_{\gamma_{p r}}(T)+\operatorname{sd}_{\gamma_{p r}}(T+e) \leq n / 2+3<n+2$.

Case 3. One of $x$ and $y$ belongs to $V\left(T_{1}\right)$, and the other does not belong to $V\left(T_{1}\right)$.

Assume, without a loss of generality, that $x \in V\left(T_{1}\right)$ and $y \notin V\left(T_{1}\right)$. By our earlier assumption, $x$ is not a stem. We distinguish two subcases.

Subcase 3.1. $x$ is a leaf of $T_{1}$.

Assume without a loss of generality that $x=v_{1}$. If $\operatorname{deg}_{T}\left(v_{3}\right)=2$ or $y$ is a leaf, then it follows from Proposition 6 that $\operatorname{sd}_{\gamma_{p r}}(T+e) \leq 4$, and the result follows from Proposition 11. Let $\operatorname{deg}_{T}\left(v_{3}\right) \geq 3$, and $y$ is not a leaf. By our earlier assumption, $y$ is not a stem, and hence each component of $(T+e)-y$ has at least two vertices. Note that the component of $(T+e)-y$ containing $v_{3}$ has at least five vertices and, thus, $\operatorname{deg}_{T+e}(y)<$ $n / 2-1$. Let $T^{\prime}$ be obtained from $T+e$ by subdividing the edges $v_{3} v_{2}, v_{2} v_{1}$, and $v_{1} y$ by the subdivision vertices $x_{1}, x_{2}$, and $x_{3}$, respectively, and all edges incident to $y$ in $T$. We show that $\gamma_{p r}\left(T^{\prime}\right)>\gamma_{p r}(T+e)$. Let $P$ be a $\gamma_{p r}\left(T^{\prime}\right)$-set, $F^{\prime}$ be the set of subdivided edges incident to $y$ whose subdivision vertices are in $P$ and let $T_{1}$ be obtained from $T+e$ by subdividing the edges in $F^{\prime}$.

First, we assume that $y \in P$ and $y$ is paired with $x_{3}$. Then, clearly $\mid P \cap\left\{x_{1}, x_{2}, v_{1}, v_{2}\right\} \geq$ 2. We may assume that $v_{3} \in P$, otherwise $u_{2}, u_{2}^{\prime} \in P$, and hence we may consider $\left(P-\left\{u_{2}^{\prime}\right\}\right) \cup\left\{v_{3}\right\}$ as a $\gamma_{p r}\left(T^{\prime}\right)$-set. If $v_{3}$ is paired with a vertex other than $x_{1}$, then $P-$ 
$\left\{x_{1}, x_{2}, v_{2}, v_{1}\right\}$ is a PD-set of $T_{1}$ with cardinality of less than $P$. If $v_{3}$ is paired with $x_{1}$, then $\left|P \cap\left\{v_{2}, v_{1}, x_{2}\right\}\right| \geq 2$ and $\left(P-\left\{x_{1}, x_{2}, v_{1}, v_{2}\right\}\right) \cup\left\{v_{2}\right\}$ is also a PD-set of $T_{1}$ with cardinality of less than $P$.

Second, assume that $y \in P$ and $y$ is paired with a subdivision vertex other than $x_{3}$. Then, $\left|P \cap\left\{x_{1}, x_{2}, v_{1}, v_{2}, x_{3}\right\}\right| \geq 2$. As above, we may assume that $v_{3} \in P$. If $v_{3}$ is paired with a vertex other than $x_{1}$, then $P-\left\{x_{1}, x_{2}, v_{1}, v_{2}, x_{3}\right\}$ is a PD-set of $T_{1}$ with cardinality of less than $P$. If $v_{3}$ is paired with $x_{1}$, then $u_{2}, u_{2}^{\prime} \in P$ and hence $P-\left\{u_{2}^{\prime}, x_{1}, x_{2}, v_{1}, v_{2}, x_{3}\right\}$ is a PD-set of $T_{2}$ obtained from $T$ by subdividing the edges in $F^{\prime}-\{e\}$, with cardinality of less than $P$.

Finally, we assume that $y \notin P$. Then, $y$ must be dominated by a subdivision vertex. As above, we may assume that $v_{3} \in P$. We consider the following.

(3.1.1) $x_{3} \in P$.

Then, $x_{3}$ and $v_{1}$ are partners and to paired-dominate $v_{2}$, we must have $\left|P \cap\left\{x_{1}, x_{2}, v_{2}, v_{3}\right\}\right| \geq$ 2. If $v_{3}$ is paired with $x_{1}$, then $u_{2}, u_{2}^{\prime} \in P$, and hence $\left(P-\left\{u_{2}^{\prime}, x_{1}, x_{2}, x_{3}, v_{1}, v_{2}\right\}\right) \cup$ $\left\{v_{1}, v_{2}\right\}$ is a PD-set of $T_{1}$ with cardinality of less than $P$. If $v_{3}$ is paired with a vertex other than $x_{1}$, then $\left|P \cap\left\{x_{1}, x_{2}, v_{2}\right\}\right| \geq 2$, and hence $\left(P-\left\{x_{1}, x_{2}, x_{3}, v_{1}, v_{2}\right\}\right) \cup\left\{v_{1}, v_{2}\right\}$ is a PD-set of $T_{2}$ with cardinality of less than $P$.

(3.1.2) $x_{3} \notin P$.

Then, we must have $v_{1}, x_{2} \in P$. Let $w_{y z} \in P$ be a subdivision vertex that dominates $y$, and let $T_{3}$ be obtained from $T+e$ by subdividing the edges $F^{\prime} \backslash\{y z\}$. If $v_{3}$ is paired with $x_{1}$, then $u_{2}, u_{2}^{\prime} \in P$, and hence $\left(P-\left\{u_{2}^{\prime}, x_{1}, x_{2}, x_{3}, v_{1}, v_{2}, w_{y z}\right\}\right) \cup\{y\}$ is a PD-set of $T_{2}$ with cardinality of less than $P$. If $v_{3}$ is paired with a vertex other than $x_{1}$, then $\left(P-\left\{x_{1}, x_{2}, x_{3}, v_{1}, v_{2}, w_{y z}\right\}\right) \cup\{y\}$ is also a PD-set of $T_{3}$ with cardinality of less than $P$.

In all cases, it follows from Proposition 1 that $\gamma_{p r}\left(T^{\prime}\right)>\gamma_{p r}\left(T_{i}\right) \geq \gamma_{p r}(T+e)$ for each $i \in\{1,2,3\}$. Therefore, we have $\operatorname{sd}_{\gamma_{p r}}(T+e) \leq \operatorname{deg}_{T+e}(y)+2<n / 2+1$. It follows from Proposition 11 that $\operatorname{sd}_{\gamma_{p r}}(T)+\operatorname{sd}_{\gamma_{p r}}(T+e)<n+1$.

Subcase 3.2. $x=v_{3}$.

By assumption, $y$ is not a support vertex. We consider the following situations.

(3.2.1) $\operatorname{deg}(y)=1$.

Let $w_{1}$ be the stem of $y$ and $w_{2}$ be the parent of $w_{1}$. Let $T^{\prime}$ be obtained from $T+e$ by subdividing the edges $v_{3} v_{4}, v_{3} y, y w_{1}$, and $w_{1} w_{2}$ with the vertices $y_{1}, y_{2}, y_{3}, y_{4}$, respectively, and the edge $v_{3} u_{i}$ with vertex $x_{i}$ for each $i$. Let $F$ be the set of all subdivided edges. Note that by the choice of the diametral path $v_{1} v_{2} \cdots v_{k}$, it is not difficult to check that $|F| \leq n / 2+2$. We show that $\gamma_{p r}\left(T^{\prime}\right)>\gamma_{p r}(T+e)$. Let $P$ be a $\gamma_{p r}\left(T^{\prime}\right)$-set. To paired-dominate $v_{1}$, we must have $\left|P \cap\left\{x_{1}, v_{1}, v_{2}\right\}\right| \geq 2$.

If $v_{3} \in P$ and $v_{3}$ is the partner of $x_{1}$, then $\left(P-\left\{x_{1}, v_{1}, v_{2}\right\}\right) \cup\left\{v_{2}\right\}$ is a PD-set of $T_{3}$ obtained from $T+e$ by subdividing the edges in $F \backslash\left\{v_{2} v_{3}\right\}$ with cardinality of less than $P$. If $v_{3} \in P$ and $v_{3}$ is the partner of $w_{v_{3} z}\left(\neq x_{1}\right)$, then $\left(P-\left\{x_{1}, v_{1}, v_{2}, w_{v_{3} z}\right\}\right) \cup$ $\left\{v_{2}\right\}$ is a PD-set of $T_{4}$ obtained from $T+e$ by subdividing the edge in $F \backslash\left\{v_{3} v_{2}, v_{3} z\right\}$ with cardinality of less than $P$.

In the following, we may assume that $v_{3} \notin P$. To paired-dominate $v_{1}$, we may assume that $x_{1}, v_{2} \in P$, and, to paired-dominate $y_{2}$, we must have $y \in P$. First, we assume that $y$ and $y_{2}$ are partners. If $y_{3} \in P$, then $\left(P-\left\{y_{2}, y, x_{1}\right\}\right) \cup\left\{v_{3}\right\}$ is a PD-set of $T_{5}$ obtained from $T+e$ by subdividing the edges in $F \backslash\left\{v_{3} y, v_{2} v_{3}\right\}$ with cardinality of less than $P$.

If $y_{3} \notin P$, then $\left(P-\left\{y_{2}, y, x_{1}\right\}\right) \cup\left\{v_{3}\right\}$ is a PD-set of $T_{6}$ obtained from $T+e$ by subdividing the edges in $F \backslash\left\{v_{3} y, v_{2} v_{3}, y w_{1}\right\}$ with cardinality of less than $P$. Second, we assume that $y$ and $y_{3}$ are partners. If $y_{4} \notin P$, then $w_{2} \in P$ and $\left(P-\left\{y_{1} y_{3}, x_{1}\right\}\right) \cup\left\{v_{3}\right\}$, is a PD-set of $T_{7}$ obtained from $T+e$ by subdividing the edges in $F \backslash\left\{y w_{1}, v_{3} y, v_{3} v_{2}\right\}$ with cardinality of less than $P$. If $y_{4} \in P$, then $\left(P-\left\{y, y_{3}, x_{1}\right\}\right) \cup\left\{v_{3}\right\}$ is also a PD-set of $T_{7}$ with cardinality of less than $P$. 
In all cases, it follows from Proposition 1 that $\gamma_{p r}\left(T^{\prime}\right)>\gamma_{p r}\left(T_{i}\right) \geq \gamma_{p r}(T+e)$ for each $i \in\{3,4, \ldots, 7\}$. Therefore, we have $\operatorname{sd}_{\gamma_{p r}}(T+e) \leq|F| \leq n / 2+2$. It follows from Proposition 11 that $\operatorname{sd}_{\gamma_{p r}}(T)+\operatorname{sd}_{\gamma_{p r}}(T+e) \leq n+2$.

(3.2.2) There is a path $y y_{1} y_{2}$ in $T$ satisfying that $\operatorname{deg}\left(y_{2}\right)=1$ and $\operatorname{deg}\left(y_{1}\right)=2$.

Let $T^{\prime}$ be obtained from $T+e$ by subdividing the edge $v_{3} u_{i}$ with vertex $x_{i}$ for each $i$, the edges $v_{3} v_{4}, e$, and $y y_{1}$ with the vertices $z_{1}, z_{2}$, and $z_{3}$, respectively, and the other edges incident to $y$. Let $F$ be the set of all subdivided edges. We note that $|F| \leq n / 2+2$. Let $P$ be a $\gamma_{p r}\left(T^{\prime}\right)$-set. To paired-dominate $v_{1}$ and $y_{2}$, we must have $\left|P \cap\left\{x_{1}, v_{1}, v_{2}\right\}\right| \geq 2$ and $\left|P \cap\left\{y_{1}, y_{2}, z_{3}\right\}\right| \geq 2$. To dominate $z_{2}$, we must have $y \in P$ or $v_{3} \in P$.

First, assume that $y \in P$. If $y$ is paired with $z_{3}$, then $y_{1}, y_{2} \in P$ and $P-\left\{y_{2}, z_{3}\right\}$ is a PD-set of $T_{8}$ obtained from $T+e$ by subdividing the edges $F-\left\{y y_{1}\right\}$ with cardinality of less than $P$. If $y$ is paired with $z_{2}$, then $\left(P-\left\{y_{1}, y_{2}, z_{2}, z_{3}\right\}\right) \cup\left\{y_{1}\right\}$ is a PD-set of $T_{9}$ obtained from $T+e$ by subdividing the edges $F-\left\{y y_{1}, e\right\}$ with cardinality of less than $P$. If $y$ is partner with a subdivision vertex $w_{y z} \notin\left\{z_{2}, z_{3}\right\}$, then $\left(P-\left\{y_{1}, y_{2}, z_{3}, w_{y z}\right\}\right) \cup\left\{y_{1}\right\}$ is a PD-set of $T_{10}$ obtained from $T+e$ by subdividing the edges $F-\left\{y z, y y_{1}\right\}$ with cardinality of less than $P$.

Second, assume that $v_{3} \in P$. If $v_{3}$ is paired with a subdivision vertex $w_{v_{3} z} \neq x_{1}$, then $\left(P-\left\{v_{1}, v_{2}, x_{1}, w_{v_{3}}\right\}\right) \cup\left\{v_{2}\right\}$ is a PD-set of $T_{11}$ obtained from $T+e$ by subdividing the edges $F-\left\{v_{3} z, v_{3} v_{2}\right\}$ with cardinality of less than $P$. If $v_{3}$ is paired with $x_{1}$, then $\left.\left(P-\left\{x_{1}, v_{1}, v_{2}\right\}\right\}\right) \cup\left\{v_{2}\right\}$ is a PD-set of $T_{12}$ obtained from $T+e$ by subdividing the edges $F-\left\{v_{3} v_{2}\right\}$ with cardinality of less than $P$.

In all cases, it follows from Proposition 1 that $\gamma_{p r}\left(T^{\prime}\right)>\gamma_{p r}\left(T_{i}\right) \geq \gamma_{p r}(T+e)$ for each $i \in\{8,9, \ldots, 12\}$. Therefore, we have $\operatorname{sd}_{\gamma_{p r}}(T+e) \leq|F| \leq n / 2+2$. It follows from Proposition 11 that $\operatorname{sd}_{\gamma_{p r}}(T)+\operatorname{sd}_{\gamma_{p r}}(T+e) \leq n+2$.

(3.2.3) There is no path $y y_{1} y_{2}$ in $T$ with $\operatorname{deg}\left(y_{2}\right)=1$ and $\operatorname{deg}\left(y_{1}\right)=2$.

Let $w_{1} w_{2} \cdots w_{m}$ be the shortest $\left(w_{1}, w_{m}\right)$-path in $T$ such that $w_{1}$ is a leaf of $T$ and $w_{m}=y$. By our assumption, $T$ has no strong stem. Thus, we have $m \geq 4$. Let $T_{1}^{\prime}$ be the component of $T-\left\{w_{3} w_{4}\right\}$ containing $w_{1}$. Note that $x, y \notin V\left(T_{1}^{\prime}\right)$. Now, using the argument applied in Case 1, we can see that $\operatorname{sd}_{\gamma_{p r}}(T)+\operatorname{sd}_{\gamma_{p r}}(T+e) \leq n+2$.

This completes the proof.

\section{Conclusions}

The main objective of this paper was to study the paired-domination subdivision number $\operatorname{sd}_{\gamma_{p r}}(G)$ of a graph $G$ defined to be the minimum number of edges that must be subdivided (where each edge in $G$ can be subdivided at most once) in order to increase the paired-domination number of $G$. We focused on trees and proved that, for any tree $T \neq P_{5}$ of order $n \geq 3$ and any edge $e \notin E(T), \operatorname{sd}_{\gamma_{p r}}(T)+\operatorname{sd}_{\gamma_{p r}}(T+e) \leq n+2$. As a consequence of this study, we pose the following conjecture.

Conjecture 1. Let $G$ be a connected graph of order $n \geq 6$. Then, $\operatorname{sd}_{\gamma_{p r}}(G)+\operatorname{sd}_{\gamma_{p r}}(G+e) \leq$ $n+2$.

Author Contributions: G.H. and S.M.S. contributed to the supervision, methodology, validation, project administration, and formal analysis. S.W., R.K., and H.K. contribute to the investigation, resources, some computations, and wrote the initial draft of the paper, which was investigated and approved by G.H., S.W., and S.M.S. who wrote the final draft. All authors have read and agreed to the published version of the manuscript.

Funding: This study was supported by the National Natural Science Foundation of China (Nos. 12061007, 11861011), the Natural Science Found of Fujian Province (No. 2020J01844), and the Open Project Program of Research Center of Data Science, Technology, and Applications, Minjiang University, China. 
Institutional Review Board Statement: Not applicable.

Informed Consent Statement: Not applicable.

Data Availability Statement: Not applicable.

Conflicts of Interest: The authors declare no conflict of interest.

\section{References}

1. Haynes, T.W.; Slater, P.J. Paired-domination in graphs. Networks 1998, 32, 199-206. [CrossRef]

2. Chellali, M.; Haynes, T.W. Total and paired-domination in trees. AKCE Int. J. Graphs. Combin. 2004, 1, 69-75.

3. Favaron, O.; Karami, H.; Sheikholeslami, S.M. Paired-domination number of a graph and its complement. Discrete Math. 2008, 308, 6601-6605. [CrossRef]

4. Haynes, T.W.; Henning, M.A. Paired-domination game played in graphs. Commun. Comb. Optim. 2019, 5, 79-94.

5. Kang, L.; Sohn, M.Y.; Cheng, T.C.E. Paired-domination in inflated graphs. Theoret. Comput. Sci. 2004, 320, 485-494. [CrossRef]

6. Proffitt, K.E.; Haynes, T.W.; Slater, P.J. Paired-domination in grid graphs. Congr. Numer. 2001, 150, 161-172.

7. Desormeaux, W.J.; Haynes, T.W.; Henning, M.A. Paired-domination in graphs. In Topics in Domination in Graphs; Haynes, T.W., Hedetniemi, S.T., Henning, M.A., Eds.; Springer International Publishing: Berlin, Germany, 2020.

8. Velammal, S. Studies in Graph Theory: Covering, Independence, Domination and Related Topics. Ph.D. Thesis, Manonmaniam Sundaranar University, Tirunelveli, India, 1997.

9. Amjadi, J. Total Roman domination subdivision number in graphs. Commun. Comb. Optim. 2020, 5, 157-168.

10. Avella-Alaminos, D.; Dettlaff, M.; Lemańska, M.; Zuazua, R. Total domination multisubdivision number of a graph. Discuss. Math. Graph Theory 2015, 35, 315-327. [CrossRef]

11. Dettlaff, M.; Kosari, S.; Lemańska, M.; Sheikholeslami, S.M. The convex domination subdivision number of a graph. Commun. Comb. Optim. 2016, 1, 43-56. [CrossRef]

12. Dettlaff, M.; Raczek, J.; Topp, J. Domination subdivision and domination multisubdivision numbers of graphs. Discuss. Math. Graph Theory 2019, 39, 829-839. [CrossRef]

13. Favaron, O.; Karami, H.; Sheikholeslami, S.M. Connected domination subdivision numbers of graphs. Util. Math. 2008, 77, 101-111.

14. Fvaron, O.; Karami, H.; Sheikholeslami, S.M. Disprove of a conjecture the domination subdivision number of a graph. Graphs Combin 2008, 24, 309-312. [CrossRef]

15. Meddah, N.; Blidia, M.; Chellali, M. On the 2-independence subdivision number of graphs. Commun. Comb. Optim. 2021, in press.

16. Favaron, O.; Karami, H.; Sheikholeslami, S.M. Paired-domination subdivision numbers of graphs. Graphs Combin. 2009, 25, 503-512. [CrossRef]

17. Amjadi, J.; Chellali, M. Complexity of the paired domination subdivision problem. 2020, submitted.

18. Egawa, Y.; Furuya, M.; Takatou, M. Upper bounds on the paired domination subdivision number of a graph. Graphs Combin. 2013, 29, 843-856. [CrossRef]

19. Hao, G.; Sheikholeslami, S.M.; Chellali, M.; Khoeilar, R.; Karami, H. On the paired-domination subdivision number of a graph. Mathematics 2021, 9, 439. [CrossRef]

20. Shao, Z.; Sheikholeslami, S.M.; Chellali, M.; Khoeilar, R.; Karami, H. A proof of a conjecture on the paired-domination subdivision number. submitted.

21. Qiang, X.; Kosari, S.; Shao, Z.; Sheikholeslami, S.M.; Chellali, M.; Karami, H. A note on the paired-domination subdivision number of trees. Mathematics 2021, 9, 181. [CrossRef] 